\title{
Impact of minor amounts of hydroperoxides on rhodium- catalyzed hydroformylation of long-chain olefins
}

\author{
M. Gerlach, ${ }^{a}$ D. Abdul Wajid, ${ }^{b}$ L. Hilfert, ${ }^{c}$ F. T. Edelmann, ${ }^{c}$ A. Seidel-Morgenstern, ${ }^{a, b}$ \\ and C. Hamel ${ }^{a, d}$
a Otto von Guericke University Magdeburg, Institute of Process Engineering, Universitätsplatz 2, 39104 Magdeburg, Germany; E-Mail: martin.gerlach@ovgu.de
${ }^{\mathrm{b}}$ Max Planck Institute for Dynamics of Complex Technical Systems Magdeburg, Sandtorstr. 1, 39106 Magdeburg, Germany
c Otto von Guericke University Magdeburg, Institute of Chemistry, Universitätsplatz 2, 39104 Magdeburg, Germany
${ }^{d}$ Anhalt University of Applied Science, Processing Engineering, Bernburger Str. 55, 06366 Köthen, Germany

The effect of minor amounts of impurities on the course of chemical reactions is often overlooked. Analyzing commercial 1-dodecene feeds, hydroperoxides were identified as critical impurities. The influence of varying hydroperoxide concentrations in olefin feeds was systematically investigated studying experimentally the rhodium-catalyzed hydroformylation using a diphosphite ligand. A significant loss of naldehyde selectivity and linear to branched ratio $(1 / b)$ was observed for increasing hydroperoxide concentrations. Feeding of additional ligand and/or purification of 1-dodecene restored the catalyst performance.

\section{Introduction}

The analysis of reaction mechanisms in homogeneous catalysis is usually done assuming ideal conditions, in particular purified substrates and solvents without impurities that could eventually mask the catalytic mechanism. Only for these ideal conditions microkinetic modelling can be successfully applied. Predictive models can then be parameterized, integrating all relevant or rate determining steps in the catalytic cycle [1]. In contrast, the chemical industry is facing non-ideal conditions. The entire exclusion of impurities, not required to carry out the target reactions, is difficult, expensive and often not feasible. Such impurities can lead to a loss of catalyst due to decomposition reactions and/or a reduction of selectivity and yield.

Deviations from ideality, also known in heterogeneous catalysis, could lead to limited transferability of derived kinetic models developed in laboratory to industrially realized processes [2]. In this context, the well-known and in large scale performed rhodium-catalyzed hydroformylation of olefins using phosphite ligands exemplifies the need for detailed studies on catalyst stability. Modified rhodium-catalysts using bulky diphosphite ligands were proven to be highly active in hydroformylation leading to high yields of the desired linear $n$-aldehyde and high regioselectivity in terms of $I / b$ ratio [3]. However, less active and selective but much cheaper rhodium $/ \mathrm{PPh}_{3}$, rhodium/TPPTS or even unmodified rhodium catalysts are applied industrially [4]. Phosphites are used as antioxidants to stabilize polymers during fabrication and storage and are thus prone to decomposition by oxidation, e.g. through peroxide [5]. As traces of oxygen and oxygenates 
are difficult to avoid in the industrial process additional feeding of ligand could be necessary to attain catalyst activity and selectivity [6]. The stability of homogeneous catalysts and ligands for hydroformylation has been reviewed by Börner and Franke and by van Leeuwen and Chadwick $[3 a, 4 b]$. Recently, the stability of mono- and diphosphite ligands against hydrolysis in the rhodium-catalyzed hydroformylation was studied by Zhang for industrially relevant conditions [7]. However, no systematic investigations were reported so far regarding the influence of peroxides on the rhodium-catalyzed hydroformylation of olefins using diphosphite ligands. Our efforts are further motivated by observed discrepancies in using a kinetic model for the rhodium/BiPhePhos-catalyzed hydroformylation of 1-dodecene (Fig. 1) not considering the presence of feed impurities [8].

\section{Characterization of four olefin feeds}

Prior to kinetic investigations, different commercial supplied samples of 1dodecene (A...D) were analyzed for the presence and quantity of hydroperoxides in the feed. Therefore, the impurities in the feeds were purified by vacuum distillation ( $p=5 \mathrm{mbar}, T=80^{\circ} \mathrm{C}$ ) in the bottom. The presence of three different hydroperoxides was validated by ${ }^{1} \mathrm{H}$ and ${ }^{13} \mathrm{C}$ NMR spectroscopy and different correlation experiments $(\mathrm{H}, \mathrm{H}-\mathrm{COSY}, \mathrm{HSQC}$, $\mathrm{HMBC}$ ). In addition, hydroperoxides were found to be the dominant impurity species in the C12 olefin feeds common with two branched dodecene species. For fast and distinct quantification a procedure was adapted from literature utilizing the hydroperoxide induced oxidation reaction of $\mathrm{Fe}^{2+}$ to $\mathrm{Fe}^{3+}$ in acidic media [9] $\mathrm{Fe}^{3+}$ forms a complex with xylenol orange that exhibits characteristic extinction at 560 and $590 \mathrm{~nm}$. Quantitative analysis of hydroperoxide concentration in various 1dodecene feeds from different supplied samples was done using UV/VIS spectroscopy. The results are summarized in Tab. 1 . In the following the term 'peroxides' will be used for hydroperoxides. The peroxide concentration in the different 1-dodecene feeds $\mathbf{A}$ to $\mathbf{D}$ varied significantly from $<0.01$ to $0.26 \mathrm{~mol} \%$ (Tab. 1, Entry $1-4$ ). For typical reaction conditions, these peroxide concentrations in the feed corresponded to a peroxide/ligand ratio of up to 7.9 [8b]. The reasons for these differences of peroxide concentrations in different feeds could be oxygen contamination in the production and purification process as well as during storage and transport [10]. The purification of $\mathbf{A}$ by simple vacuum distillation significantly reduced the concentration of peroxides to $0.01 \mathrm{~mol}-\%$ (Tab. 1, Entry 8) $\neq$. 


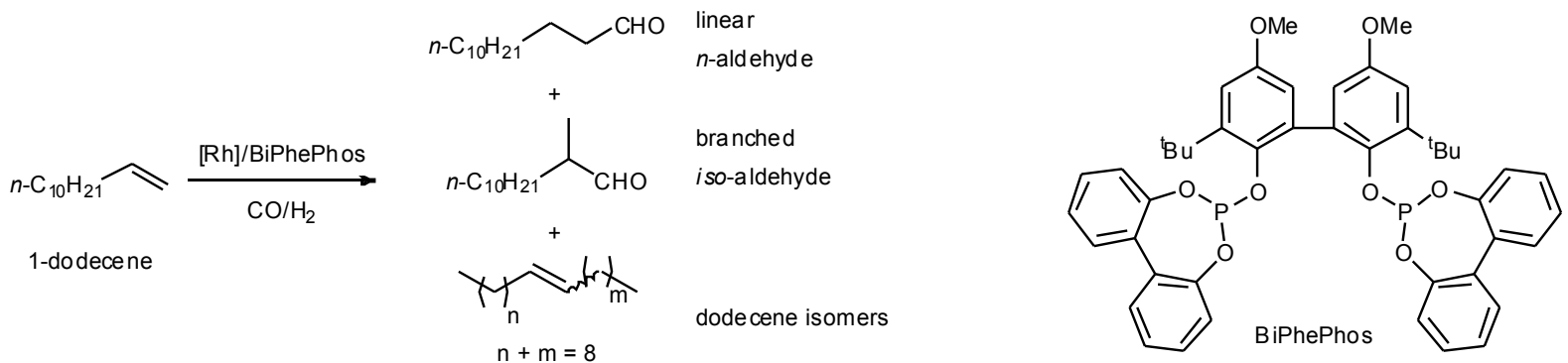

Fig. 1 Hydroformylation of 1-dodecene using Rh/BiPhePhos catalyst.

\section{Performance comparison}

Hydroformylation experiments of 1-dodecene were performed in a stainless steel reactor at 30 bar $\mathrm{CO} / \mathrm{H}_{2}(1: 1)$ and $105{ }^{\circ} \mathrm{C}$ using $\mathrm{Rh}$ (acac) $(\mathrm{CO})_{2}$ as precatalyst and BiPhePhos as ligand. Decane and $\mathrm{N}, \mathrm{N}$ dimethylformamide (DMF) were used as solvents to constitute a thermomorphic solvents system [11b]. The main results for the four olefin feeds $\mathbf{A}$ to $\mathbf{D}$, differing in their peroxide quantity, are summarized in Tab. 1 (Entry 1 - 4). The catalyst activity, expressed as the turnover frequency, TOF $=n_{\text {1-olefin }} / n_{\text {catalys } /} / t_{\text {reaction, }}$ did not show a significant change with the peroxide concentration which was also reflected in the high conversion ( $X$ $>$

99\%) of 1-dodecene. The calculated values for supplied samples C and D are in good agreement with reported results for rhodium/BiPhePhoscatalyzed hydroformylation of 1-

Tab. 1 Screening of different 1-dodecene feeds in Rh/BiPhePhos-catalyzed hydroformylation ${ }^{a}$

\begin{tabular}{|c|c|c|c|c|c|c|c|c|c|c|}
\hline $\begin{array}{l}\text { Entr } \\
y\end{array}$ & $\begin{array}{l}\text { Supplie } \\
r\end{array}$ & $\begin{array}{l}\text { Peroxides } \\
\text { in feed }\end{array}$ & $\begin{array}{l}\text { Perox } \\
\text { ide/li } \\
\text { gand }\end{array}$ & $\begin{array}{l}\text { Liga } \\
\text { nd } \\
\text { /Rh } \\
\end{array}$ & $\begin{array}{l}\text { Conve } \\
\text { rsion }\end{array}$ & $\begin{array}{l}\text { TO } \\
F\end{array}$ & Select & vity & $1 / b^{b}$ & $S T Y_{l i g}$ \\
\hline & & $\begin{array}{l}\text { (mol- } \\
\%)\end{array}$ & $(-)$ & $(-)$ & $(\%)$ & $\begin{array}{l}\left(\mathrm{h}^{-}\right. \\
\left.{ }^{1}\right)\end{array}$ & $\begin{array}{l}\mathrm{n}- \\
\text { aldeh } \\
\text { yde } \\
(\%)\end{array}$ & $\begin{array}{l}\text { iso- } \\
\text { olefi } \\
\text { ns } \\
(\%)\end{array}$ & $(-)$ & $\begin{array}{l}\left(\mathrm{kg} \mathrm{m}^{-3} \mathrm{~h}^{-1}\right. \\
\left.\text { gligand }^{-1}\right)\end{array}$ \\
\hline 1 & A & 0.26 & 7.9 & 3.3 & 99 & $\begin{array}{l}62 \\
70\end{array}$ & 50 & 45 & $\begin{array}{l}\text { 93: } \\
7\end{array}$ & 237 \\
\hline 2 & B & 0.16 & 4.8 & 3.3 & 99 & $\begin{array}{l}63 \\
40\end{array}$ & 67 & 25 & $\begin{array}{l}94: \\
6\end{array}$ & 327 \\
\hline 3 & C & 0.01 & 0.3 & 3.3 & 99 & $\begin{array}{l}64 \\
10\end{array}$ & 81 & 14 & $\begin{array}{l}98: \\
2\end{array}$ & 396 \\
\hline 4 & D & $<0.01$ & 0.1 & 3.3 & 99 & $\begin{array}{l}64 \\
10\end{array}$ & 81 & 14 & $\begin{array}{l}98: \\
2\end{array}$ & 396 \\
\hline 5 & $A^{c}$ & 0.01 & - & 0 & 84 & $\begin{array}{l}53 \\
40\end{array}$ & 8 & 88 & $\begin{array}{l}73: \\
27\end{array}$ & $2^{d}$ \\
\hline 6 & $\mathbf{A}$ & 0.26 & 2.6 & 10 & 99 & $\begin{array}{l}62 \\
20\end{array}$ & 61 & 33 & $\begin{array}{l}94: \\
6\end{array}$ & 97 \\
\hline 7 & $\mathbf{A}$ & 0.26 & 0.5 & 50 & 97 & $\begin{array}{l}61 \\
30\end{array}$ & 88 & 8 & $\begin{array}{l}99: \\
1\end{array}$ & 137 \\
\hline
\end{tabular}




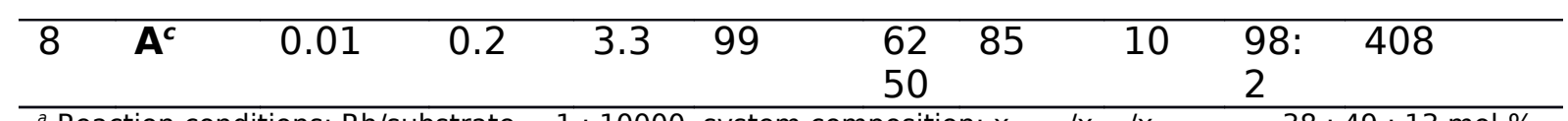

${ }^{a}$ Reaction conditions: Rh/substrate $=1: 10000$, system composition: $\mathrm{X}_{\text {decane }} / \mathrm{X}_{\mathrm{DMF}} / \mathrm{x}_{1 \text {-dodecene }}=38: 49: 13 \mathrm{~mol}-\%$, $\mathrm{V}_{\text {liquid }}=900 \mathrm{~mL}, \mathrm{~T}=105{ }^{\circ} \mathrm{C} \mathrm{K}, \mathrm{p}\left(\mathrm{CO} / \mathrm{H}_{2}=1: 1\right)=30 \mathrm{bar}$, $\mathrm{t}_{\text {reaction }}=90 \mathrm{~min}$. ${ }^{b}$ Ratio of $n$ - to iso-aldehyde in all aldehydes. ${ }^{c}$ Purified sample of $\mathbf{A}$ by vacuum distillation $\left(\mathrm{p}=5 \mathrm{mbar}, \mathrm{T}=80^{\circ} \mathrm{C}\right) .{ }^{d} \mathrm{STY}=m_{n \text {-aldehyde }} / \mathrm{N}_{\text {liquid }}$ $/ t_{\text {reaction. }}$.

dodecene [8b,11]. In contrast, the selectivity towards the n-aldehyde significantly decreased for supplied samples A and B with a peroxide/ligand ratio $>0.3$. A significant change in selectivity towards an increased isomerization of 1 -dodecene to iso-dodecenes was observed. Additionally, the regioselectivity of the catalyst in terms of the $1 / b$ ratio of aldehydes was decreased resulting in higher production of undesired isoaldehyde. Thus, the ligand specific space time yield of $\mathrm{n}$-aldehyde, $S T Y_{\text {lig }}=$ $m_{\text {n-aldehyde }} / N_{\text {liquid }} / t_{\text {reaction }} / m_{\text {ligand }}$, was clearly decreased from 396 to $237 \mathrm{~kg} \mathrm{~m}^{-3} \mathrm{~h}^{-}$ ${ }^{1} \mathrm{~g}_{\text {ligand }}{ }^{-1}$ for increasing peroxide/ligand ratios of 0.3 to 7.9 , respectively. In addition, the hydroformylation using an unmodified rhodium catalyst (Tab. 1 , Entry 5) revealed the lowest catalyst performance parameters. The $n$ aldehyde selectivity, $I / b$ ratio and the STY are clearly decreased as compared to the ligand modified hydroformylation experiments.

Further analysis of the investigated reaction kinetics (Fig. 2a) confirmed a significant effect of peroxides on the selectivity and regioselectivity versus time. For supplied samples $\mathbf{C}$ and $\mathbf{D}$ with low peroxide feed concentrations high initial isomerization rates were found, which has already been reported for the rhodium/BiPhePhos catalyst leading to low n-aldehyde selectivities at the beginning of the experiments $[8,12]$. As the hydroformylation of 1-dodecene proceeded, the $n$-aldehyde selectivity increased until the end of the experiment. High isomerization rates were found to reduce the production of iso-aldehyde and thus, the regioselectivity in terms of the $I / b$ ratio remained almost constant at a high level [4b]. In contrast, for supplied samples $\mathbf{A}$ and $\mathbf{B}$ with high peroxide concentrations low initial isomerization rates were observed leading to an increased $n$-aldehyde selectivity at the beginning of the experiments. Subsequently, an increase of isomerization rates and a loss of n-aldehyde selectivity was observed until the end of experiment. The point of catalyst performance change was indicated in the $1 / 6$ ratio, which decreased simultaneously. The duration until the catalyst performance change takes place was prolonged for supplied sample B with a lower peroxide concentration relative to $\mathbf{A}$.

The above described observations clearly indicate a change in the catalytic system and therefore a degradation of the diphosphite ligand BiPhePhos through peroxides can be proposed. The time-dependent decomposition by oxidation could lead to different mono- and diphosphite ligands [3a,5a]. Thus, new catalytic species emerge over the course of reaction with different intrinsic activities and selectivities (Fig. 3). As a result, the measured kinetics are a superposition of these different interacting catalytic species competing for the metal center and the 
substrate with their different intrinsic reaction kinetics. Therefore, model discrepancies arise when the decomposition of ligand reaches a critical level and rhodium/BiPhePhos is not the dominant catalytic species.

a)
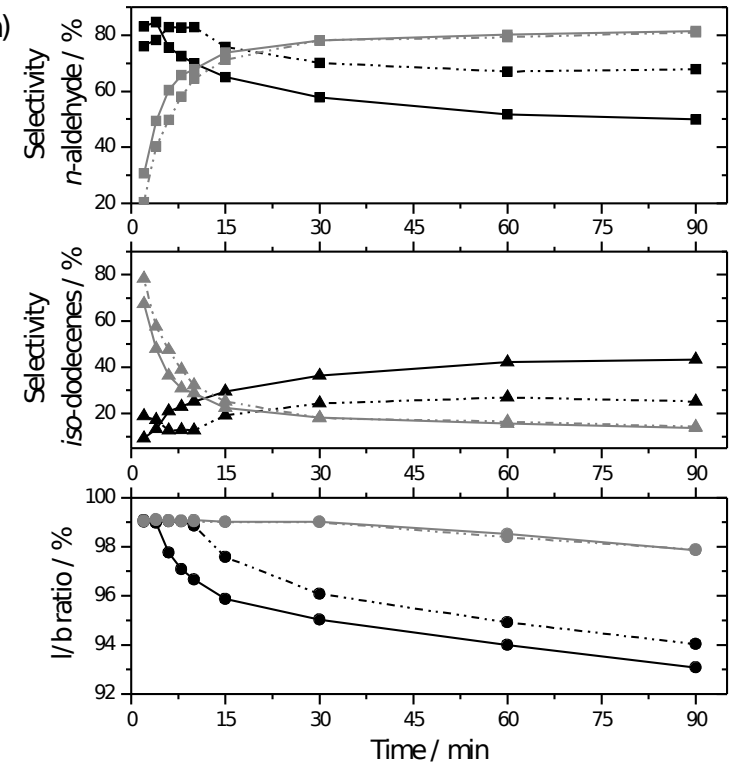

\begin{tabular}{c} 
Suppliers \\
\hline A \\
- B \\
C \\
\hline D
\end{tabular} b)
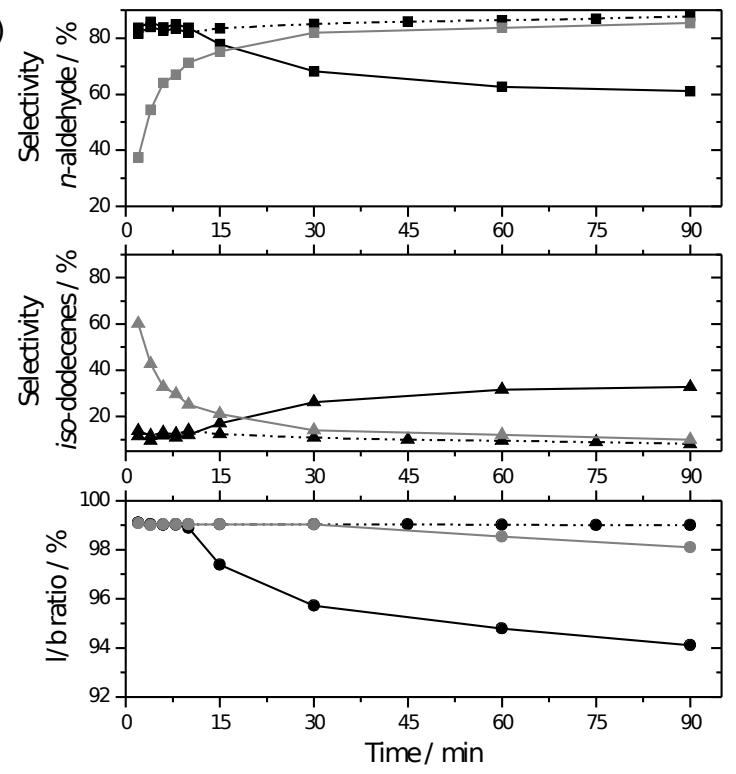

Suppliers Peroxide/Ligand Ligand/Rh

Fig. 2 Rh/BiPhePhos-catalyzed hydroformylation of 1-dodecene: Influence of hydroperoxides on selectivity for $n$-aldehyde and iso-dodecenes and $1 / \mathrm{b}$ ratio over time for (a) four different 1-dodecene supplied samples with varying hydroperoxide quantity, (b) one specific supplied sample with decreased peroxide/ligand ratio by additional feeding of ligand and purification of 1-dodecene by vacuum distillation. (for reaction conditions see Tab. 1)

The assumption of ligand decomposition has been validated in additional hydroformylation experiments where the feed of supplied sample $\mathbf{A}$ was used and the peroxide/ligand ratio was decreased (Fig. 2b) 抽. At first, an additional amount of ligand was added to the catalyst solution prior to the experiment increasing the ligand/Rh ratio (Tab. 1, Entry 6 - 7). For a ligand/Rh ratio of 10 , the change in catalyst performance was similar to supplied sample B. No change of catalyst performance was observed for a ligand/Rh ratio of 50 leading to the highest $I / b$ ratio as well as $n$-aldehyde selectivity of $99: 1$ and $88 \%$, respectively. However, STYlig was clearly reduced with a high amount of ligand used to attain the catalyst performance. In addition, the feed was purified by vacuum distillation (Tab. 1, Entry 8) prior to the experiment. Similar to the results of supplied samples $\mathbf{C}$ and $\mathbf{D}$, no significant change in catalyst performance was observed. The peroxide to ligand ratio was decreased to 0.2 by reducing the absolute concentration of peroxides in the feed leading to high values

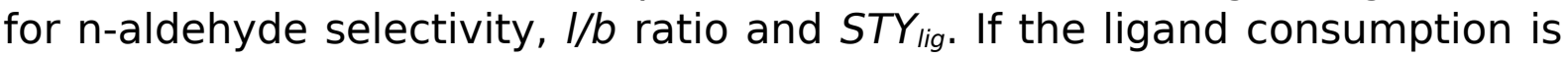
an important economical factor, decreasing the absolute amount of peroxides is the most feasible scenario. 
The comparison of the transients (Fig. $2 b$ ) revealed a difference for the run with the distilled substrate in which the selectivity for the n-aldehyde increased over time. This trend was also observed for supplied samples $\mathbf{C}$ and D (Fig. 2a) with a low quantity of peroxides. These results clearly indicate an inhibition of the isomerization of 1-dodecene due to decomposition products of the ligand BiPhePhos. Hereby, an important requirement for the $\beta$ - $\mathrm{H}$-elimination is a vacant site at the rhodium center, which could be blocked by decomposition products of the ligand [13].

\section{Conclusions}

A significant influence of hydroperoxides, typically present in commercially applied feed streams, was observed during the rhodium-catalyzed hydroformylation of 1-dodecene. The identification and quantification of hydroperoxides causing the de-composition of the diphosphite ligand BiPhePhos were performed by NMR and UV/VIS spectroscopy, respectively. For higher concentrations of hydroperoxides in the feed, a significant gradual loss of $n$-aldehyde selectivity and regioselectivity in terms of $I / b$ ratio was quantified. Consequently, detailed knowledge about decomposition kinetics of catalyst/ligand complexes is required to derive reliable kinetic models. The integration of additional catalytic cycles according to the emerging catalytic species will lead to even more complex models. Improved reactor operation was achieved by adjusting a peroxide/ligand ratio and the absolute peroxide concentration as important parameters. Decreasing the peroxide/ligand ratio through feeding of additional ligand or purification of the feed restored the catalyst performance. More extended spectroscopic investigations to study the decomposition of ligands due to the presence of hydroperoxide are currently underway. The usage of scavengers like epoxides and amines to stabilize the ligand will be addressed in future work $[6 a, 14,15]$.

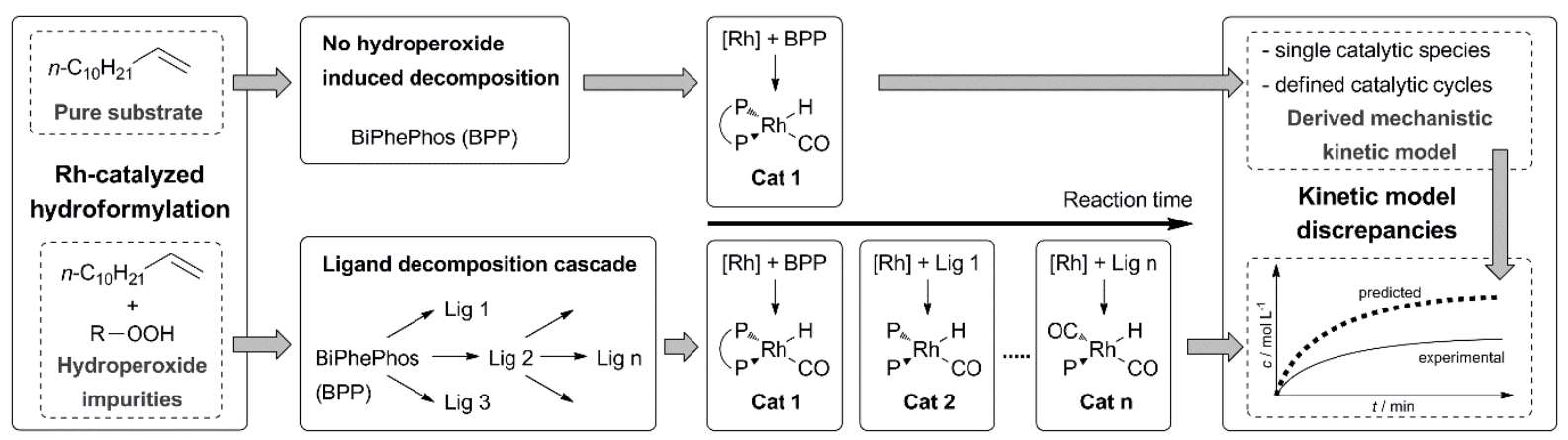

Fig. 3 Proposed hydroperoxide induced, time-dependent decomposition of ligand BiPhePhos (BPP) leading to different catalytic species and kinetic model discrepancies.

\section{Acknowledgements}

This work is part of the Collaborative Research Center/Transregio 63 "Integrated Chemical Processes in Liquid Multiphase Systems". The authors thank the German Research Foundation (DFG) for the financial 
support. We also thank Umicore AG \& Co. KG for the donation of the rhodium precursor $\mathrm{Rh}(\mathrm{acac})(\mathrm{CO})_{2}$.

\section{Notes and references}

¥ The purification of olefin feeds can be improved by refluxing the olefin with sodium or diphosphite ligand prior to distillation to remove last traces of hydroperoxides.

㧊 Ligand decomposition was also indicated using ${ }^{31} \mathrm{P}$ NMR spectroscopy. Details can be found in the ESI. 